\title{
Gold nanoparticles: testbeds for engineered protein-particle interactions
}

\author{
"Taken together, we have come a long way in understanding and engineering \\ particle-protein interactions, with a large proportion of the insights being provided \\ by gold nanoparticles."
}

Keywords: enzymatic activity $\bullet$ functionalized gold nanoparticles $\bullet$ nonfouling properties - protein corona $\bullet$ protein stability $\bullet$ self-assembled monolayers $\bullet$ stealth nanoparticles

Controlling the interactions of nanoparticles with biosystems is crucial for using these materials in diagnostic, imaging and therapeutic applications. Interfacing nanoparticles with proteins is an important issue for each of these applications. In diagnostic applications, for example, the ability to recognize proteins provides access to sensor systems for quantifying biomarkers and profiling complex biofluids [1]. Protein recognition is similarly important in therapeutic and imaging applications, where the interaction with specific markers is a desirable feature. Equally essential for all of these cases is the ability to turn off interactions (i.e., creating 'stealth' nanoparticles) [2]. On the sensing side, noninteracting coatings can be used to enhance specificity and prevent biofouling. With diagnostics and imaging agents, stealth coatings can be used to help evade immune responses - especially since neutralizing nanoscale objects (e.g., bacteria and viruses) is a central goal of the immune system.

Along with the promise of engineered nanoparticle-protein interactions come multiple challenges. First, there is an enormous spectrum of protein sizes, charges, hydrophobicities and other parameters that need to be considered when engineering the interface [3]. Second, proteins are at very high concentrations in biofluids (e.g., $\sim 1 \mathrm{mM}$ in human serum). This high concentration means that even rather weak interactions will complicate desired recognition events. Third, and perhaps most daunting of all, is that protein structures are highly dynamic and can be readily distorted and even denatured through binding events [4].

Gold nanoparticles (AuNPs) provide a particularly useful platform for engineering and studying biointeractions [5]. The surfaces of these particles can be readily and stably tuned using thiol-based ligands that bind strongly to the gold surface. For smaller particles ( $\leq 6-8-\mathrm{nm}$ core diameter), wellformed monolayers can be generated, providing chemical control of the surface through the generation of homogeneous and mixed monolayer particles [6]. Larger particles can be similarly functionalized; however, their surface structure is by nature less regular, featuring ligands and ionic species at the surface. Next, elemental gold is nontoxic, meaning that the lessons we learn with AuNPs can be directly translated to real-world applications [7]. Finally, our experience has shown that AuNPs are frankly the easiest particles to make in a stable form in useful quantities.

The creation of noninteracting particles is a challenge that has continued over decades of research [8]. This long and difficult problem is due at least in part to the fact that 'noninteracting' is an ideal, not a reality. When thinking of materials in complex biofluids, it helps to think of a very crowded party - one where you will continually bump into people. The key to navigating both parties and serum is to minimize the interaction time, avoiding sticky and undesirable interactions that would result in aggregation.

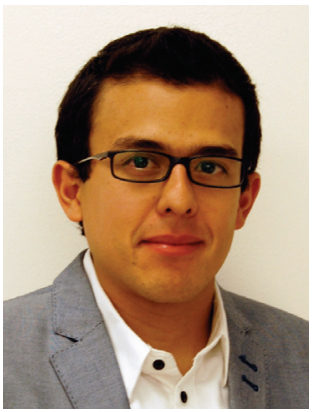

Daniel F Moyano Department of Chemistry, University of Massachusetts, 710 North Pleasant Street, Amherst, MA 01003, USA

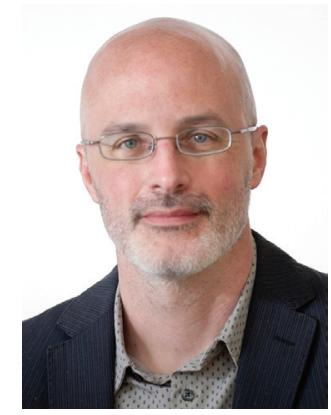

Vincent M Rotello Author for correspondence: Department of Chemistry, University of Massachusetts, 710 North Pleasant Street, Amherst, MA 01003, USA rotello@chem.umass.edu 
The traditional way of generating stealth particles is through the use of PEG ligands [2,9]. Although PEG has been employed widely both in vitro and in vivo, there are two limitations to this approach. First, PEG is uncharged, making it challenging to engineer the desired electrostatic and hydrophobic interactions [10]. This issue has been addressed with smaller AuNPs that require commensurately short PEG units. Small AuNPs (2-6-nm core diameter) can be functionalized with short PEG units (e.g., tetraethylene glycol) that allow functionality to be synthetically placed at the ligand terminus [11]. Other particles can be functionalized with longer PEG chains in mixed monolayer AuNPs.

While PEG functionalization has been widely employed with nanomaterials and works well for ex vivo applications, recent studies have shown that PEG chains of different sizes will elicit the formation of antibodies [12]. This immune response is actually quite a problem, resulting in complement activation that can generate a range of physiological reactions, including anaphylactic shock. Fortunately, selfcomplementary cation-anion pairs (i.e., zwitterions [9]) have been shown to have excellent resistance to protein fouling, and have recently been shown to be nonimmunogenic [13].

\section{"...recent studies have shown that PEG chains of different sizes will elicit the formation of antibodies."}

As mentioned above, nanoparticle-protein interactions will always be present in serum and in cells. A complicating feature of these interactions is that proteins will tend to alter their structure in order to optimize their interactions with surfaces. This denaturation process converts reversible protein-particle interactions into irreversible states, generating a protein coat that has been termed the 'protein corona' [14]. This corona can mask desired interactions, as well as foster immune response and induce aggregation. While many researchers feel that the generation of this corona is inevitable, appropriate engineering of the particle monolayer in order to foster reversible protein-particle interactions provides a path to avoiding irreversible fouling interactions with the concomitant formation of permanent coronas [15].

Clearly, generating particles that bind proteins reversibly would be helpful for delivery. Controlled interactions of this sort are a requirement in applications in which delivery of the active protein/enzyme is essential and targeting functionalities may be masked by proteins that have been adsorbed in a nonreversible fashion [16]. Reversible binding is also crucial for sensing, where denaturation results in sensor fouling and inactivity.
Initial studies of protein-particle interactions using amphiphilic particles resulted in protein denaturation at the particle surface, generating the widely held belief that denaturation is inevitable at particle surfaces. This assumption is quite incorrect - nanoparticles have been engineered to stabilize the protein structure, maintaining and even enhancing activity [17]. AuNPs have been particularly successful in this regard in applications requiring the maintenance of protein structure, including sensing, delivery and even 'green chemistry' strategies using biocatalysts.

What are the secrets to generating reversible protein-particle interactions? First, size is an important parameter. Smaller particles are better at preventing denaturation for a variety of reasons, particularly the radius of curvature [18]. Second, amphiphilicity must be handled very carefully - there is a strong energetic push for hydrophobic protein residues to interact with hydrophobic surfaces. That is not to say that all greasiness is bad, as AuNPs with hydrophobic head groups were found to maintain protein structure better than purely hydrophilic analogs [19]. Finally, ligand flexibility is crucial - if the particle cannot adapt to the protein, the protein will adapt (i.e., denature) to the particle.

Small AuNPs provide an excellent platform for providing all of the above attributes. In addition to their demonstrated utility in applications, the nature of this AuNP-protein interaction has been explored using calorimetry [20]. This study demonstrated that 2-nm core AuNPs with appropriate surfaces effectively mimic protein-protein interactions, making these particles, in essence, artificial proteins.

Taken together, we have come a long way in understanding and engineering particle-protein interactions, with a large proportion of the insights being provided by AuNPs. We have some answers regarding how to generate noninteracting particles, and some demonstrated successes in generating reversible particle-protein interactions. However, all of these insights relate to specific systems. What remains is to convert the one-off insights of individual studies into general engineering principles. Once this goal has been achieved, we will have a truly 'plug-and-play' playbook for creating protein-nanoparticle bioconjugates.

\section{Financial \& competing interests disclosure}

VM Rotello acknowledges support from the NIH (EB014277 and GM077173). The authors have no other relevant affiliations or financial involvement with any organization or entity with a financial interest in or financial conflict with the subject matter or materials discussed in the manuscript apart from those disclosed.

No writing assistance was utilized in the production of this manuscript. 


\section{References}

1 Saha K, Agasti SS, Kim C, Li X, Rotello VM. Gold nanoparticles in chemical and biological sensing. Chem. Rev. 112, 2739-2779 (2012).

2 Jokerst JV, Lobovkina T, Zare RN, Gambhir SS. Nanoparticle PEGylation for imaging and therapy. Nanomedicine 6, 715-728 (2011).

3 De M, Miranda OR, Rana S, Rotello VM. Size and geometry dependent protein-nanoparticle self-assembly. Chem. Commun. 2157-2159 (2009).

4 Nel AE, Mädler L, Velegol D et al. Understanding biophysicochemical interactions at the nano-bio interface. Nat. Mater. 8, 543-557 (2009).

5 Weintraub K. Biomedicine: the new gold standard. Nature 495, S14-S16 (2013).

6 Moyano DF, Rotello VM. Nano meets biology: structure and function at the nanoparticle interface. Langmuir 27, 10376-10385 (2011).

7 Alkilany AM, Murphy CJ. Toxicity and cellular uptake of gold nanoparticles: what we have learned so far? J. Nanopart. Res. 12, 2313-2333 (2010).

8 Hoffman AS. The origins and evolution of 'controlled' drug delivery systems. J. Control. Release 132, 153-163 (2008).

9 Cao Z, Jiang S. Super-hydrophilic zwitterionic poly(carboxybetaine) and amphiphilic non-ionic poly(ethylene glycol) for stealth nanoparticles. Nano Today 7 , 404-413 (2012).

10 Sekiguchi S, Niikura K, Matsuo Y, Ijiro K. Hydrophilic gold nanoparticles adaptable for hydrophobic solvents. Langmuir 28, 5503-5507 (2012).

11 You C-C, De M, Rotello VM. Contrasting effects of exterior and interior hydrophobic moieties in the complexation of amino acid functionalized gold clusters with $\alpha$-chymotrypsin. Org. Lett. 7, 5685-5688 (2005).

12 Wang XY, Ishida T, Kiwada H. Anti-PEG IgM elicited by injection of liposomes is involved in the enhanced blood clearance of a subsequent dose of PEGylated liposomes. J. Control. Release 119, 236-244 (2007).

13 Yang W, Liu S, Bai T et al. Poly(carboxybetaine) nanomaterials enable long circulation and prevent polymerspecific antibody production. Nano Today 9, 10-16 (2014).

14 Lynch I, Dawson KA. Protein-nanoparticle interactions. Nano Today 3, 40-47 (2008).

15 Casals E, Puntes VF. Inorganic nanoparticle biomolecular corona: formation, evolution and biological impact. Nanomedicine 7, 1917-1930 (2012).

16 Liu R, Kay BK, Jiang S, Chen S. Nanoparticle delivery: targeting and nonspecific binding. MRS Bull. 34, 432-440 (2009).

17 You C-C, Agasti SS, De M, Knapp MJ, Rotello VM. Modulation of the catalytic behavior of $\alpha$-chymotrypsin at monolayer-protected nanoparticle surfaces. J. Am. Chem. Soc. 128, 14612-14618 (2006).

18 Shang W, Nuffer JH, Muñiz-Papandrea VA et al. Cytochrome $\mathrm{C}$ on silica nanoparticles: influence of nanoparticle size on protein structure, stability, and activity. Small 5, 470-476 (2009).

19 You C-C, De M, Han G, Rotello VM. Tunable inhibition and denaturation of $\alpha$-chymotrypsin with amino acid-functionalized gold nanoparticles. J. Am. Chem. Soc. 127, 12873-12881 (2005).

20 De M, You C-C, Srivastava S, Rotello VM. Biomimetic interactions of proteins with functionalized nanoparticles: a thermodynamic study. J. Am. Chem. Soc. 129, 10747-10753 (2007). 